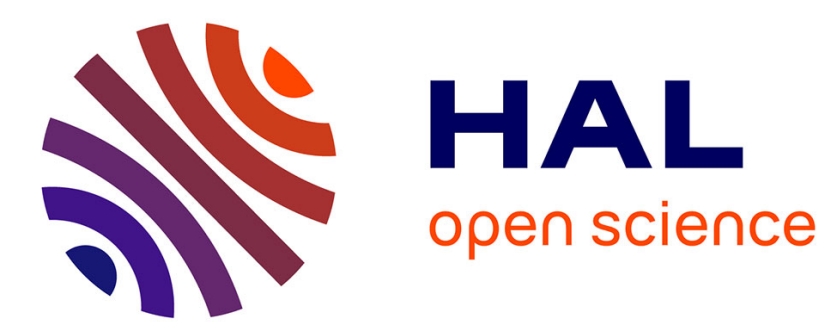

\title{
X-ray scattering functions of fractal structures : comparison between simulations and experiments
}

\author{
M.A.V. Axelos, D. Tchoubar, R. Jullien
}

\section{To cite this version:}

M.A.V. Axelos, D. Tchoubar, R. Jullien. X-ray scattering functions of fractal structures: comparison between simulations and experiments. Journal de Physique, 1986, 47 (10), pp.1843-1847. 10.1051/jphys:0198600470100184300 . jpa-00210380

\section{HAL Id: jpa-00210380 https://hal.science/jpa-00210380}

Submitted on 1 Jan 1986

HAL is a multi-disciplinary open access archive for the deposit and dissemination of scientific research documents, whether they are published or not. The documents may come from teaching and research institutions in France or abroad, or from public or private research centers.
L'archive ouverte pluridisciplinaire HAL, est destinée au dépôt et à la diffusion de documents scientifiques de niveau recherche, publiés ou non, émanant des établissements d'enseignement et de recherche français ou étrangers, des laboratoires publics ou privés. 
Classification

Physics Abstracts

$61.10-61.40 \mathrm{~K}-82.70 \mathrm{D}$

\title{
X-ray scattering functions of fractal structures : comparison between simulations and experiments
}

\author{
M. A. V. Axelos $\left({ }^{*}\right)$, D. Tchoubar and R. Jullien $\left({ }^{\dagger}\right)$ \\ Laboratoire de Cristallographie, C.N.R.S., U.A. 810, Université d'Orléans, U.F.R.-Faculté des Sciences, \\ B.P. 6759, Rue de Chartres, 45067 Orléans Cedex 2, France \\ ${ }^{\dagger}$ ) Laboratoire de Physique des Solides, Bât 510, Université de Paris-Sud, Centre d’Orsay, 91405 Orsay \\ Cedex, France
}

(Reçu le 11 mars 1986, révisé le 29 mai, accepté le 26 juin 1986)

\begin{abstract}
Résumé. - Les spectres de diffusion aux petits angles produits par deux solutions d'hydroxyde d'aluminium $\mathrm{Al}(\mathrm{OH})_{x}(\operatorname{avec} x=2,5$ et $x=2,6)$ sont comparées quantitativement à ceux déduits à partir de simulations numériques relatives aux modèles d'agrégation par collage d'amas $(\mathrm{Cl}-\mathrm{Cl})$. Nous montrons que si l'échantillon $x=2,6$ est bien représenté par le modèle standard $\mathrm{Cl}-\mathrm{Cl} 3 \mathrm{~d}$, par contre les agrégats formés à $x=2,5$ correspondent à un modèle $3 \mathrm{~d}$ récemment développé, dit « par collage de pointes » qui fait intervenir la polarisabilité des amas.
\end{abstract}

\begin{abstract}
Small angle X-ray scattering (S.A.X.S.) functions of aluminum hydroxide aggregates $\mathrm{Al}(\mathrm{OH})_{x}$, are quantitatively compared with the ones obtained from numerical simulations on cluster-cluster ( $\mathrm{Cl}-\mathrm{Cl})$ aggregation models. While the $x=2.6$ case is fitted by the standard $3 \mathrm{~d}$ model, the $x=2.5$ case can be fitted by a newly developed « tip-to-tip » $3 \mathrm{~d}$ model, which considers cluster polarizability.
\end{abstract}

\section{Introduction.}

$3 d$ aggregation experiments have been performed on aluminum hydroxide species $\mathrm{Al}(\mathrm{OH})_{x}[1,2]$. In the $x=2.5$ case [1], the analysis of the small angle $\mathrm{X}$-ray scattering (S.A.X.S.) gives a fractal dimension $D=1.45$ smaller than the $3 d$-values of the different theoretical models which have been introduced to describe the geometry of clusters obtained by aggregation of particles $[3,4]$. Indeed, the clustering of cluster model $(\mathrm{Cl}-\mathrm{Cl})$, which is better adapted to aggregation of colloïds, has a $3 d$-fractal dimensions $D \sim 1.78$ [4], this value decreases to $D \sim 1.44$ in $2 d$ simulations [3].

Arguing that aluminum hydroxide subunits have anisotropic interactions due to their internal structure, we first concluded [1] to an effective $2 d \mathrm{Cl}-\mathrm{Cl}$ aggregation. Recently, an extension of $\mathrm{Cl}-\mathrm{Cl}$ : the « tip-to-tip » model (T-T), which considers smaller penetration, has been proposed [5] which leads to smaller fractal dimension : $D \sim 1.42$ and 1.28 , in $3 d$ and $2 d$ respectively. This model, which is physically justified in the case of polarizable clusters, allows us to put forward $3 d$ aluminum hydroxide resulting clusters. In this paper, we present a quantitative comparison between the experimental scattering functions $I(s)$ with the ones deduced from the exact distance distribution function of simulated finite sized clusters. Three theoretical models are

(*) Present address : I.N.R.A., Laboratoire de Physicochimie des Macromolécules, rue de la Géraudière, 44072 Nantes, France. studied: the $2 d-\mathrm{Cl}-\mathrm{Cl}$ and $3 d-\mathrm{T}-\mathrm{T}$ models are compared with $\mathrm{Al}(\mathrm{OH})_{2.5}$ clusters and $3 d-\mathrm{Cl}-\mathrm{Cl}$ is compared with $\mathrm{Al}(\mathrm{OH})_{2.6}$ which has a fractal dimension of $D \sim 1.86$ [2].

\section{Experiments.}

2.1 MATERIALS. - The colloïdal samples of aluminum hydroxide were made by partial hydrolysis of aluminum chloride solution with sodium hydroxide following a method described elsewhere [6]. The samples are characterized by the hydrolysis ratio $x$ defined as $(\mathrm{OH}) /(\mathrm{Al})$, where the $\mathrm{Al}$ concentration remains constant : $(\mathrm{Al})=0.1 \mathrm{M} / \mathrm{l}$. Previous results have shown that for $x<2.3$, the solution is a stable sol of cationic species, named $\mathrm{Al}_{13}$ polymers, which are spherical particles of $25 \AA$ diameter. When $x$ increases towards 2.6 , the decrease of the electrical charges induces aggregation phenomena : for $x=2.5$ the resulting clusters exhibit a fractal dimension of $D=1.45$, for $x=2.6$ precipitation starts to occur and $D$ is about $1.86[1,2]$.

2.2. X-RAY DATA RECORDING. - We used the synchrotron radiation of DCI storage ring of LURE (Université, Paris-Sud, Orsay) to benefit from a very highly intense X-ray beam associated with point collimation and a very performed device which allows to reach very small angles. Indeed the collected date cover the $s$-range from 0.001 to 0.06 $\AA^{-1}$ and from 0.0005 to $0.013 \AA^{-1}$, where here $s$ is the scattering vector amplitude : $s=2 \sin \theta / \lambda, 2 \theta$ the scattering angle and $\lambda$ the selected wave length (here $\lambda=1.6 \AA$ ). 
2.3 X-RAY DATA ANALYSIS. - The experimental scattering function $I(s)$ of fractal aggregates is given by :

$$
I(s)=I_{0}(s) \cdot[G(s) * F(s)]
$$

* denotes a convolution product.

$I_{0}(s)$ is the scattering function of a single $20 \AA$ diameter spherical subunit which can be reduced, in the experimental $\mathrm{s}$ range, to :

$$
I_{0}(s)=V_{0} \exp -4 \pi^{2} R g_{0}^{2} s^{2} / 3
$$

Where $V_{0}$ and $R g_{0}$ are respectively the volume and the radius of gyration of the subunit.

$G(s)$ is the interference function between these subunits, $G(s)$ scales as $s^{-D}$ between the points $s \sim 1 / 2 \pi \zeta$ and $s \sim 1 / 2 \pi r_{0}$ where $r_{0}$ is the subunit radius and $\zeta$ a characteristic length of the clusters.

$F(s)$ is the natural cut-off function of the aggregate above the characteristic distance $\zeta$.

From the exact inter-particle distance distribution function $P(r)$ calculated from the simulated finite size cluster (cf. 3), the Fourier transform allows us to obtain directly the product $G(s){ }^{*} F(s)$ without any assumption about the analytic expression of the $F(s)$ function. Then the theoretical expression of the scattering function is given by [7] :

$I(s)=I_{0}(s) \cdot\left[1+\frac{4 \pi}{V_{0}} \int_{0}^{\infty} P(r) \frac{\sin 2 \pi s r}{2 \pi s r} \mathrm{~d} r\right]$

$I(s)$ is normalized such as $I(0)=V$, the volume of the cluster, that means that the distance distribution $P(r)$ is normalized such as :

$$
\left[\begin{array}{l}
4 \pi \frac{1}{V_{0}} \int_{0}^{\infty} P(r) \mathrm{d} r=N-1 \\
\text { with } P(0)=0
\end{array}\right]
$$

$N$ being the number of subunits in the cluster.

The comparison between the theoretical and experimental curves is performed by adjusting the intensity in the range $s \geqslant 1 / 2 \pi r_{0}$, where the inter-particle interferences become negligeable and the intensity is then only characteristic of the scattering by a single subunit.

2.4. RESULTS. - S.A.X.S. experiments carried out with $x=2.5$ and the $x=2.6$ samples have shown that the aggregation of the $\mathrm{Al}_{13}$ subunits yields to fractal aggregates of dimension $D \sim 1.45$ and $D \sim 1.86$ respectively $[1,2]$. The $I(s)$ scattering functions of the two samples are depicted in a $\log _{10}-\log _{10}$ plot (Fig. 1).

One observes a clear linear part which is characteristic of fractal behaviour. In the 2.5 case one can see the departure from the linear law for $s \sim 1 / 2 \pi r_{0}$, which allows an estimation of the radius of this subunit : $r_{0} \approx 10 \AA$. Moreover, the linear extrapolation of the 2.6 curve joins the 2.5 curve at about the same $s$ value. This is consistent with the previous conclusion

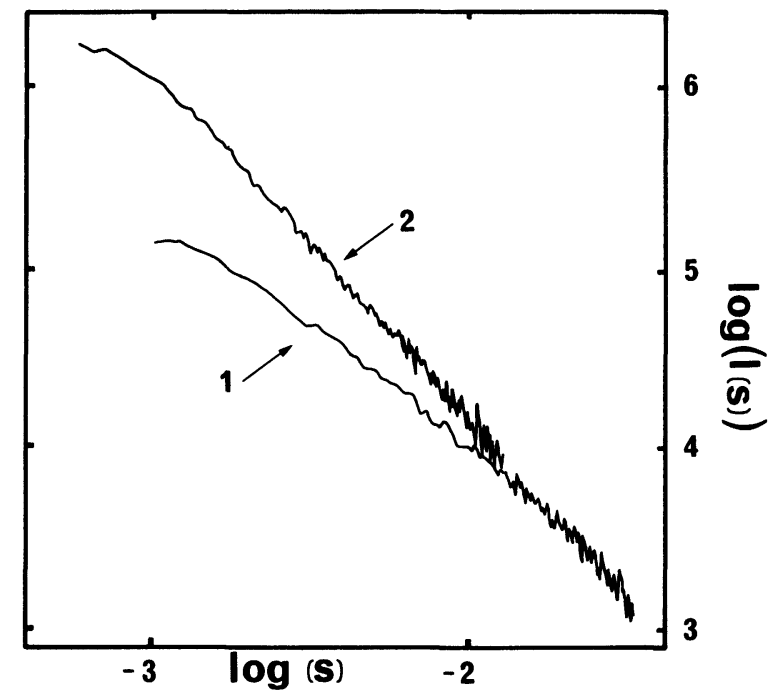

Fig. 1. - Experimental scattering functions $I(s)$ in a $\log _{10}-\log _{10}$ plot. The curves 1 and 2 correspond to $x=2.5$ and 2.6 samples, respectively.

that the subunits are the same in both samples [2]. On the other hand, from the bending of the curve for small $s$-values one can estimate the characteristic length in both cases $\zeta \approx 94 \AA$ and $140 \AA$ for $x=2.5$ and 2.6 , respectively.

\section{Simulations.}

3.1. Models. - In the $\mathrm{Cl}-\mathrm{Cl}$ model [3] clusters as well as particles, are allowed to diffuse in space and stick together when they become in contact. This model is well adapted to describe aerosol and colloïd aggregation experiments where the experimental fractal dimension $D \sim 1.75[8]$ is close to the $3 d$ simulated value (1.78). Many extensions and simplifications of this model have been introduced. In particular it has been shown that replacing brownian trajectories by linear trajectories does not affect so much the results, increasing only slightly the fractal dimension from 1.44 to 1.51 in $2 d$ and from 1.78 to 1.91 in $3 d$ [9]. Another recent extension, the T-T model considered the effect of cluster polarizability [5]. In the case of strong polarisability the clusters systematically stick by their tips leading to a smaller fractal dimension 1.42 in $3 d, 1.26$ in $2 d$ the $2 d$ value might satisfactorily account for $2 d$ aggregation experiments of silica microspheres [10].

In the simulations proposed here we have systematically used the hierarchical version of these models [11], in which successive collections of clusters of the same number of particles $2,4, \ldots 2^{n}$ are built iteratively, starting from a set of individual particles. At each step the clusters are grouped into pairs and the two clusters of the pair collide to give a cluster of the new collection. The collision process depends on the model. In the $\mathrm{Cl}-\mathrm{Cl}$ case we have considered an off-lattice version with linear trajectories [9] where the trajectory is chosen to be a random straightline in space (a new choice is done if no collision occurs). Linear trajectories have been considered here only 
for simplicity and economy reasons, knowing that the results would be very similar with the Brownian model. In the T-T case, a random direction is first chosen. The particles on each clusters of the pairs which have the largest and smallest abscissa along this direction are chosen and the clusters are translated such that these two particles become on contact, aligned along the chosen direction.

3.2. SIMULATED DISTANCE DISTRIBUTION FUNCTIONS. - In the three cases, $\mathrm{Cl}-\mathrm{Cl} 2 d, \mathrm{Cl}-\mathrm{Cl} 3 d$ and T-T $3 d$ we have generated 100 independent clusters of 64 to 1024 particles and we have directly calculated a « reduced " distance distribution function $P(x)$ for each size by simply establishing the histogram of inter-particle distances within the aggregate. For each integer $x\left(x=r / d_{0}, d_{0}=2 r_{0}\right) \widetilde{P}(x)$ is the number of distances $\left|x_{i}-x_{j}\right|$ located between $x-0.5$ and $x+0.5 \cdot \widetilde{P}(x)$ has been averaged over all the trials and divided by the number of particles such that the integral of $\widetilde{P}(x)$ is exactly the number of particle $N$. As defined here, this distribution is such as :

$$
\int \widetilde{P}(x) \mathrm{d} x=N ; \widetilde{P}(0)=1
$$

In figure 2 , we present the two $3 d$ curves, in the case of $\mathrm{Cl}-\mathrm{Cl}$ and T-T models of 1024 particles whose

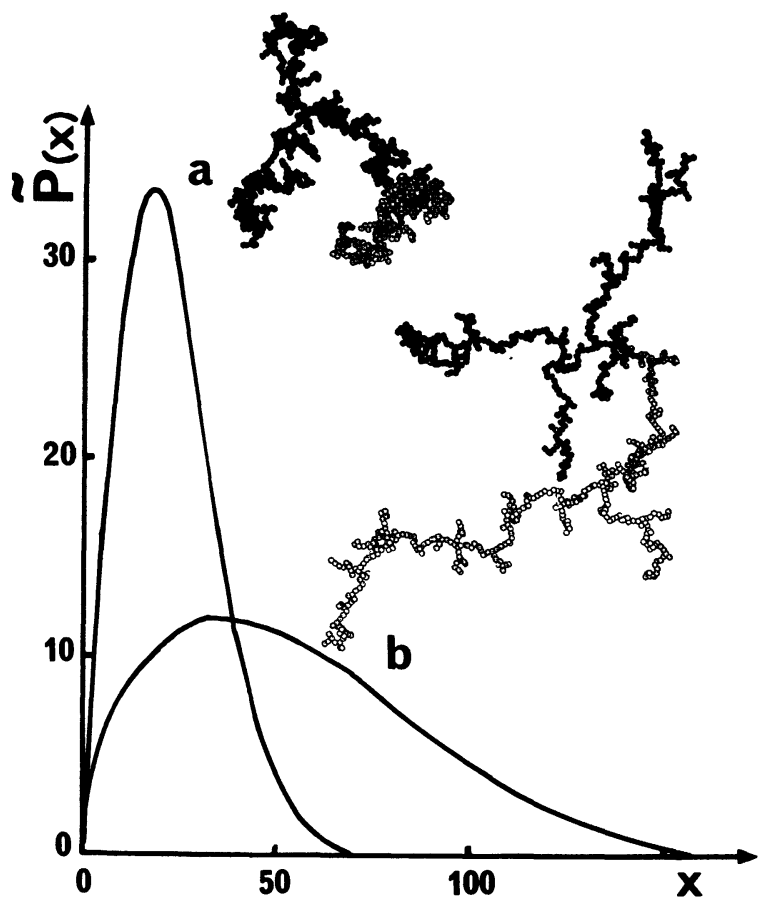

Fig. 2. - Distance distribution function $\widetilde{P}(x)$ as deduced from the simulations on $3 d$ models with the same number of particles $(N=1024)$. The curve a) corresponds to the $\mathrm{Cl}-\mathrm{Cl}$ model with linear trajectories whose fractal dimension is $D=1.90$. The curve b) corresponds to the T-T model whose fractal dimension is $D=1.43$. In each case we have given an example of typical aggregate, with the same scale, to better show the role of the fractal dimension on the size extension of the clusters. fractal dimensions are 1.9 and 1.43 respectively. The effect of the value of the fractal dimension on the overall shape of the curve is clearly seen. In the small $x$ region $\widetilde{P}(x)$ varies as $x^{D-1}$, then goes through a maximum at about $x \sim \zeta / d_{0}$ and decreases continuously up to the larger distance in the aggregate.

In figure 3 , we present the $\widetilde{P}(x)$ curves here the two models $\mathrm{T}-\mathrm{T} 3 d$ and $\mathrm{Cl}-\mathrm{Cl} 2 d$ which have almost the same fractal dimension, 1.43 and 1.50 respectively. Note that this comparison is allowed here since $\widetilde{P}(x)$ is an intrinsic function which directly measures the distance-distribution function inside the aggregate. In any space dimension, $\widetilde{P}(x)$ should behave as $x^{D-1}$ for small $x$. However, as seen in figure 3 , the two curves are remarkably close, even in the large $x$ region. This was not an obvious result $a$ priori since the region above the maximum corresponds mostly to the " active surface zone " of the clusters. where the sticking mecanism might have an influence on the distance distributions.

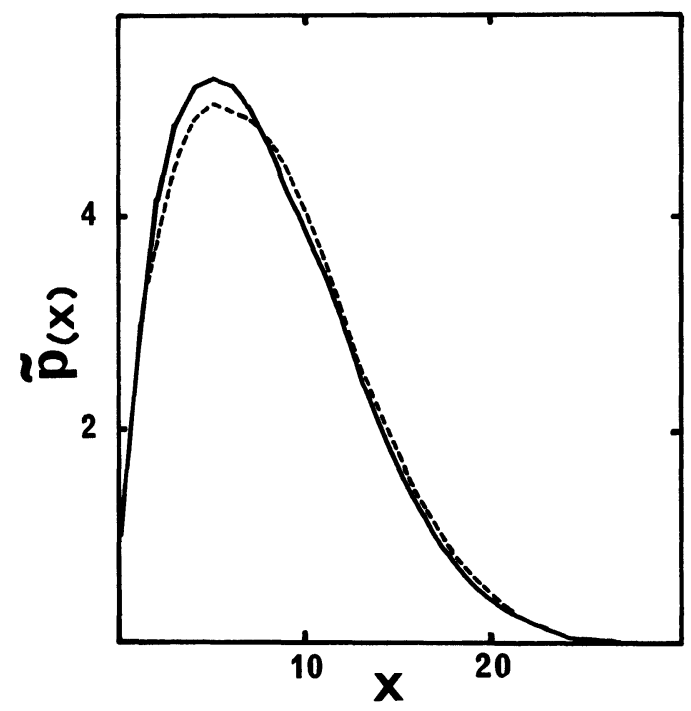

Fig. 3. - Comparison between the distance distribution functions $\widetilde{P}(x)$, as deduced from the simulations with the $3 d \mathrm{~T}$-T model $(\longrightarrow)$ and with the $2 d \mathrm{Cl}-\mathrm{Cl}$ model (----), with $N=64$ particles.

\section{Quantitative comparison between experiments} and simulations.

From the above calculated $\widetilde{P}(x)$ distribution functions, one can evaluate the theoretical $I(s)$ scattering functions through formula (2), by properly defines $P(r)$ by :

$$
P(r)=\frac{V_{0}}{4 \pi d_{0}} \widetilde{P}\left(\frac{r}{d_{0}}\right) .
$$

Note that in the case of $\mathrm{Cl}-\mathrm{Cl}-2 \mathrm{~d}$, the use of formula (2) should lead to the scattering function of randomly oriented $2 d$-cluster imbedded in a $3 d$ space, which thus can be compared with experimental $3 d$ curves. The $\widetilde{P}(x)$ curves being roughly the same for $\mathrm{Cl}-\mathrm{Cl}-$ $2 \mathrm{~d}$ and $\mathrm{T}-\mathrm{T}-3 d$, the resulting $I(s)$ would be very 
similar and thus the fit with the experimental curve cannot allows us to decide between the two models. We will thus present only the comparison of the case $x=2.5$ with the T-T-3d model.

The quantitative fit with the T-T-3d model in the case $x=2.5$ is shown in figure 4 . We recall that the only adjustable parameter is the intensity about $s \approx 1 / 2 \pi r_{0}$. The linear behaviour is well recovered in agreement with the fractal dimension $D \approx 1.45$ previously reported [1]. We must note that this $D$ value however does not represent exactly the slope observed in the figure 4 since here due to our small $\zeta / r_{0}$ value $\left(\zeta / r_{0} \approx 10\right)$ we were obliged to take into account the size effect of the subunit (1) ; only $I(s) / I(0)(s)$ would scale as $s^{-D}$. Moreover the fact that the shape of the curve above $s \sim 1 / 2 \pi r_{0}$ is well reproduced confirms, a posteriori, the choice of $I_{0}(s)$.

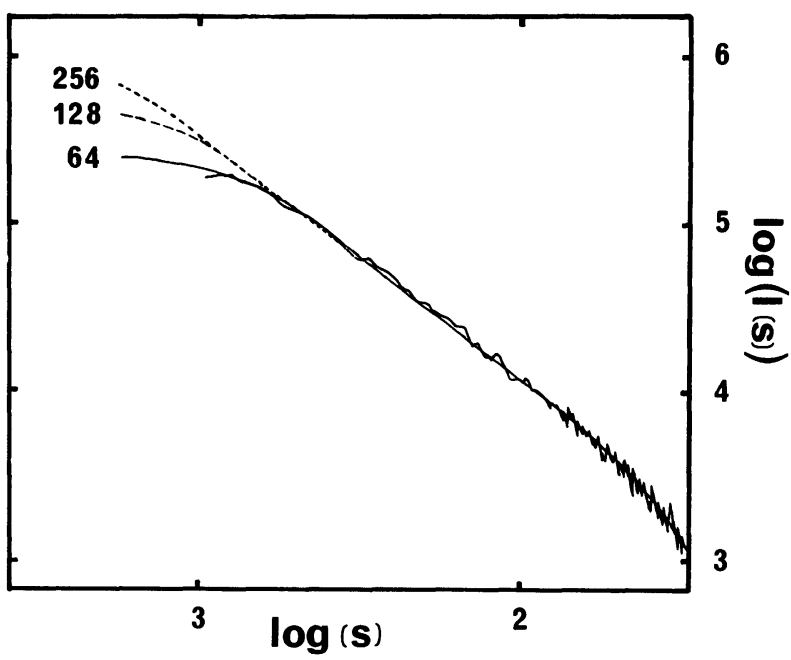

Fig. 4. - This figure shows three theoretical scattering curves $I(s)$, using the $3 d$ T-T model, with $N=64$ ( cles. The best fit for the $x=2.5$ sample is obtained with $N=64$ particles.

In figure 4 we have shown three theoretical curves corresponding to different number of particles in the aggregate. As expected only the small-s part of the curve is affected and by choosing the best fit with the experiment, one can estimate the number of particle to be $N \approx 64$.

The same kind of fit can be obtained between the $x=2.6$ experimental curve and the simulated one using $3 \mathrm{~d}-\mathrm{Cl}-\mathrm{Cl}$, as shown in figure 5 . The best fit is here obtained for $N \approx 512$ particles.

\section{Discussion.}

The fact that the $x=2.5$ experimental curve can be fitted by the $3 d$ T-T model is very satisfactory since it is not necessary to invoke a preferential orientation of the $\mathrm{Al}_{13}$ subunit to produce a $2 d$ long range aggregation as we previously claimed [1]. However,

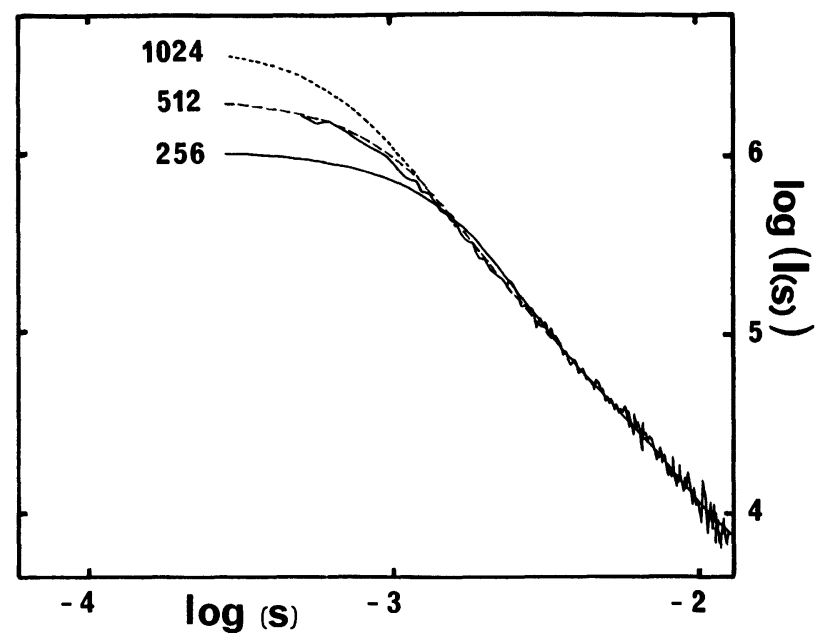

Fig. 5. - This figure shows three theoretical scattering curves $I(s)$, using the $3 d \mathrm{Cl}-\mathrm{Cl}$ model, with $N=256$ (,$- N=512$ (-----) and $N=1024$ (.......) particles. The best fit for the $x=2.6$ sample is obtained with $N=512$ particles.

the fitting alone cannot be conclusive to distinguish between $3 d \mathrm{~T}-\mathrm{T}$ and $2 d \mathrm{Cl}-\mathrm{Cl}$ since the whole $\widetilde{P}(x)$ curve seems to depend only on the fractal dimension value and not on the dimension of space and on the details of the sticking mechanism. A scattering experiment in presence of an electrical field inducing orientation of the aggregates could probably permit a distinction between the two hypothesis.

Moreover, one can develop some qualitative arguments to explain why the T-T model is more adapted to the $x=2.5$ case while the $\mathrm{Cl}-\mathrm{Cl}$ model describe the $x=2.6$ case. The $\mathrm{Al}_{13}$ subunit are positively charged and water molecules are oriented near their surface, producing a surface field. The intensity of this field is directly linked with the importance of the charge on the subunits. For $x=2.5$ the charge is strong and one can imagine that the polarization of the water molecules near the surface of the aggregates might induce a sticking mechanism like in the T$\mathrm{T}$ model. When $x$ increases the charge on the subunits decreases, the water molecule surface charges decrease also and one can imagine that the standard diffusion-sticking mechanism could be recovered as in the regular $\mathrm{Cl}-\mathrm{Cl}$ model. Moreover, one can learn, from the fits, that, with the same initial concentration of $\mathrm{Al}_{13}$ and the same ageing time for the aggregation process, the aggregates contain much more particles in the case $x=2.6$ than in the case $x=2.5$. We must remember that in the present simulations no size distribution has been considered for the clusters and the numbers of particles within the clusters must be only considered more like averaged orders of magnitude than precise numbers. The increase of the number of particles is however clear when going from $x=2.5$ to 2.6. This implies that the two models must have completely different kinetics. 
To conclude, we have been able to fit the entire experimental scattering function by using simulated clusters. This allowed a direct comparison between the different theoretical model and experiments. Further works are in progress to extend the present study.

\section{Acknowledgments}

We acknowledge support for the numerical computations from an ATP of the CNRS. Calculations were performed at CIRCE (Centre Interrégional de Calcul Electronique).

\section{References}

[1] Axelos, M., Tchoubar, D., Bottero, J. and Fiessinger, F. J. Physique, 46 (1985) 1587.

[2] Bottero, J. Y., AXelos, M. A. V., TChoubar, D., Cases, J. M., Fripiat, J. J. et Fiessinger, F., Preprint.

[3] Meakin, P., Phys. Rev. Lett. 51 (1983) 1119.

Kolb, M., Botet, R. and Jullien R., Phys. Rev. Lett. 51 (1983) 1123.

[4] Jullien, R., Kolb, M. and Botet, R., J. Physique, 45 (1984) L211.

[5] Jullien, R., Phys. Rev. Lett. 55 (1985) 1697 and J. Phys. A 19 (1986) 2129.

[6] Bottero, J. Y., CASES, J. M., Fiessinger, F. and POIRIER, J. E., Phys. Chem. 84 (1980) 2933.
[7] GUINIER, A., FOURNET, G., Small-angle scattering of $X$ rays (John-Wiley and Son) 1955.

[8] Forrest, S. and Witten, T., J. Phys. A 12 (1979) L109.

Weitz D. and Oliveira, M., Phys. Rev. Lett. 52 (1981) 1433.

[9] Ball, R. and Jullien, R., J. Physique 45 (1984) L1031.

MEAKIN, P., J. Colloid and Interface, Science 102 (1984) 505.

[10] Hurd, A. and Shaefer, D., Phys. Rev. Lett. 54 (1985) 1043.

[11] Botet, R., Jullien, R. and Kolb, J. Phys. A 17 (1984) L75. 